\title{
ÉCOPHYTOSOCIOLOGIE ET PRODUCTION LIGNEUSE
}

\author{
M. BECKER \\ Laboratoire de Botanique forestière, I. N. R. A., \\ E. N.G.R. E. F, \\ 54042 Nancy

\section{RÉSUMÉ}

La définition de stations isopotentielles sur le plan de la productivité (en qualité et en quantité) peut être envisagée à l'aide de critères variés. En plus des critères purement dendrométriques ou purement écologiques - étudiés dans d'autres exposés - la végétation spontanée peut également être souvent un instrument d'utilisation très commode. II y a des critères strictement floristiques, utilisés depuis fort longtemps dans les forêts boréales (CAJANDER, 1926). Ceux-ci paraissant mal adaptés à la complexité des forêts tempérées, il a fallu attendre la notion de "groupe écologique "s, et les travaux de DuCHaufour essentiellement, pour yoir apparaitre de véritables criteres phytoécologiques. Ces groupes écologiques (groupes d'espèces à écologie semblable) sont utiles pour la définition et la cartographie des stations forestières. Ils peuvent être élaborés à l'aide de méthodes trés variées, faisant appel à des moyens plus ou moins complexes : méthode traditionnelle * des tableaux ", méthode des groupes écologiques statistiques (GouNOT), méthodes diverses faisant appel aux analyses multivariables (composantes principales, analyse factorielle). L'analyse écologique peut, suivant les cas, précéder ou suivre l'analyse floristique. L'appréciation de la productivité peut être envisagée en deux phases : une phase qualitative lors de l'inventaire phyto-écologique préalable; une phase quantitative à l'occasion de l'étude écologique plus détaillée des types stationnels reconnus.

\section{INTRODUCTION}

La connaissance de la forêt et de ses potentialités comporte un certain nombre d'aspects, en principe complémentaires.

Dans un premier temps, il importe au forestier de savoir prévoir la production ${ }^{(1)}$ de ses massifs, en utilisant des critères qui peuvent être très divers. C"est sur ce point, objet essentiel de notre exposé, que nous reviendrons le plus longuement.

Dans un deuxième temps, se fait sentir le besoin de pouvoir expliquer la production afin, dans un troisième temps, de mieux savoir la contrôler.

En dehors de l'aspect génétique de la question, l'explication de la production, c'est-à-

1. Par production, nous entendons aussi bien l'aspect quantitatif (production en volume), que la qualité technologique des bois produits, et, le cas échéant, l'aptitude des peuplements à se régénérer naturellement. 
dire la recherche des mécanismes mis en œuvre, fait appel aux diverses spécialités de l'écologie (pédologie, bioclimatologie, étude des relations sociales...), de l'écophysiologie et de la physiologie proprement dite.

Par le travail du sol et la fertilisation, le forestier pourra alors agir sur les facteurs édaphiques, et, par une sylviculture raisonnée, sur les facteurs microclimatiques et les phénomènes de concurrence. On sait naturellement que, fort heureusement, la sylviculture n'a pas attendu que tous les mécanismes de la production soient élucidés pour commencer à imaginer et à tester des techniques culturales adaptées aux besoins du forestier praticien.

\section{LES DIVERS CRITERES DE STATION}

Ce sont eux qui doivent permettre de prévoir la production.

En tout premier lieu, et pour mémoire, il existe des critères purement dendrométriques, les indices de productivité, auxquels, à défaut de connaître la production elle-même, il est d'ailleurs nécessaire de faire appel pour étalonner les autres types de critéres. Cet aspect a été développé dans l'exposé de Dícourt.

\section{Critères floristiques}

L'utilisation de critères purement floristiques pour définir les stations repose sur le principe que la végétation est l'élément synthétisant le mieux l'ensemble des conditions de milieu. La répartition naturelle des espèces dépend en effet, de façon plus ou moins stricte, des divers facteurs écologiques.

Acquise de très longue date, puisque Korstıan déjà, en 1917, préconisait l'utilisation de la végétation naturelle pour déterminer les stations forestières, cette constatation a permis d'établir des classifications de forêts basées sur la composition floristique, soit du peuplement arborescent, soit du sous-bois, soit de l'ensemble. La productivité moyenne de chaque type ainsi reconnu peut être estimée.

Les travaux du Finlandais CAJANDER $(1926,1943)$ s'attachent plus particulièrement à cette notion, dite du type forestier, qui a d'ailleurs permis d'établir une systématique très valable des stations forestières boréales.

CAJANDER reprochait aux critères purement dendrométriques utilisés alors, de caractériser des complexes station-peuplement - ce dernier composé d'une ou de quelques essences bien précises, et soumis à un traitement donné - et non les stations elles-mêmes. De plus, sa méthode lui semblait avoir aussi l'avantage de mettre en évidence des entités naturelles, tangibles géographiquement, et non des abstractions graphiques telles que des classes de productivité.

Par définition, caractérisent un type forestier donné toutes les stations qui, lorsqu'elles portent des peuplements mûrs, ont une flore de même composition, ainsi que celles qui ne s'écartent de cette composition porentielle que sous l'influence momentanée d'un « accident » quelconque (incendie, pâturage... ). Selon les conditions écologiques du moment, chaque type comporte un certain nombre d'associations, qui se caractérisent toutes par la même fertilité potentielle. De fait, grâce à une étude de Ilvessalo (1920) en Finlande, il 
s'est avéré que la relation était excellente entre les types forestiers de CAJANDER et la productivité des stations que ces derniers avaient permis de définir; de telle sorte qu'ils purent être utilisés comme critère de fertilité dans l'établissement de tables de productivité pour la forêt boréale (mélange Pin sylvestre, Épicéa et Bouleau ; voir fig. 1).

Il faut reconnaittre que les forêts boréales conviennent tout particulièrement à la mise en œuvre de cette démarche. Ce sont des forêts naturelles très vastes, oủ les conditions écologiques ne varient que progressivement (climat, géologie), et sont, en valeur absolue, très dures pour la végétation; ceci exige de la part de l'association existante (et du peuplement forestier), une grande compétitivité qui tend à simplifier la structure du tapis végétal.

Dans les forêts plus ou moins remaniées, voire totalement artificielles, qui sont les nôtres en Europe occidentale, I'utilisation systématique d'une méthode purement floristique rencontre des difficultés certaines. La diversité écologique y est beaucoup plus importante et la flore beaucoup plus riche. Intrinsèquement, il est donc plus difficile d'y dégager les diverses unités floristiques. Mais surtout, l'influence humaine y a été beaucoup plus forte, amenant d'importantes perturbations dans la composition floristique, celle du peuplement productif, mais aussi, par contre-coup, celle du sous-bois. A la limite, dans certains cas de plantations denses de résineux, la flore naturelle disparait totalement et ne saurait donc être utilisée... Cette difficulté s'est d'ailleurs surtout manifestée en Allemagne, où les feuillus spontanés ont été, depuis deux siècles, remplacés systématiquement par des résineux (RUBNER, 1934).

L'utilisation des critères floristiques peut également s'avérer insuffisante ou difficile dans le cas des terrains dits " marginaux ", que l'on a l'intention de reboiser et dont on souhaite prévoir la productivité selon les essences susceptibles d'être introduites. Dans les friches, par exemple, la végétation n'est pas encore stabilisée et les unités floristiques, encore fluantes, sont plus difficiles à dégager. Mais, naturellement, c'est surtout I'étalonnage de la végétation vis à vis de la production potentielle qui pose des problèmes. Dans ce cas, ceci ne sera guère possible que par l'intermédiaire de critères écologiques, eux-mêmes étalonnés dans des milieux proches et boisés.

Par ailleurs, s'il est d'une utilisation particulièrement simple - à condition toutefois de ne pas être foncièrement allergique à la botanique _, l'outil que constitue la végétation nécessite quelques précautions d'emploi; en particulier, il faudra se garder d'extrapoler trop rapidement à d'autres régions, sans contrôle préalable, des résultats obtenus dans une région donnée.

\section{Critères écologiques}

L'ensemble de ces difficultés ont contribué, parallèlement à l'utilisation de critères purement floristiques, au développement de méthodes basées sur la seule étude des facteurs écologiques. Ce sont eux, effectivement, qui conditionnent directement la production ligneuse, alors que la végétation du sous-bois n'en est, en général, qu'un indice. Il est naturellement hors de notre propos de développer cet aspect, qui fait l'objet d'autres exposés. Nous voudrions seulement souligner que les variables écologiques pouvant être étudiées sont innombrables; faire un choix efficace est un problème ardu lorsque le chercheur se trouve face à une question nouvelle, et le risque peut être grand d'analyser à grand frais des variables qui ne sont en fait que simplement corrélées avec les vraies variables explicatives, elles-mêmes non mesurées. Seule l'expérimentation peut alors permettre de trancher. 


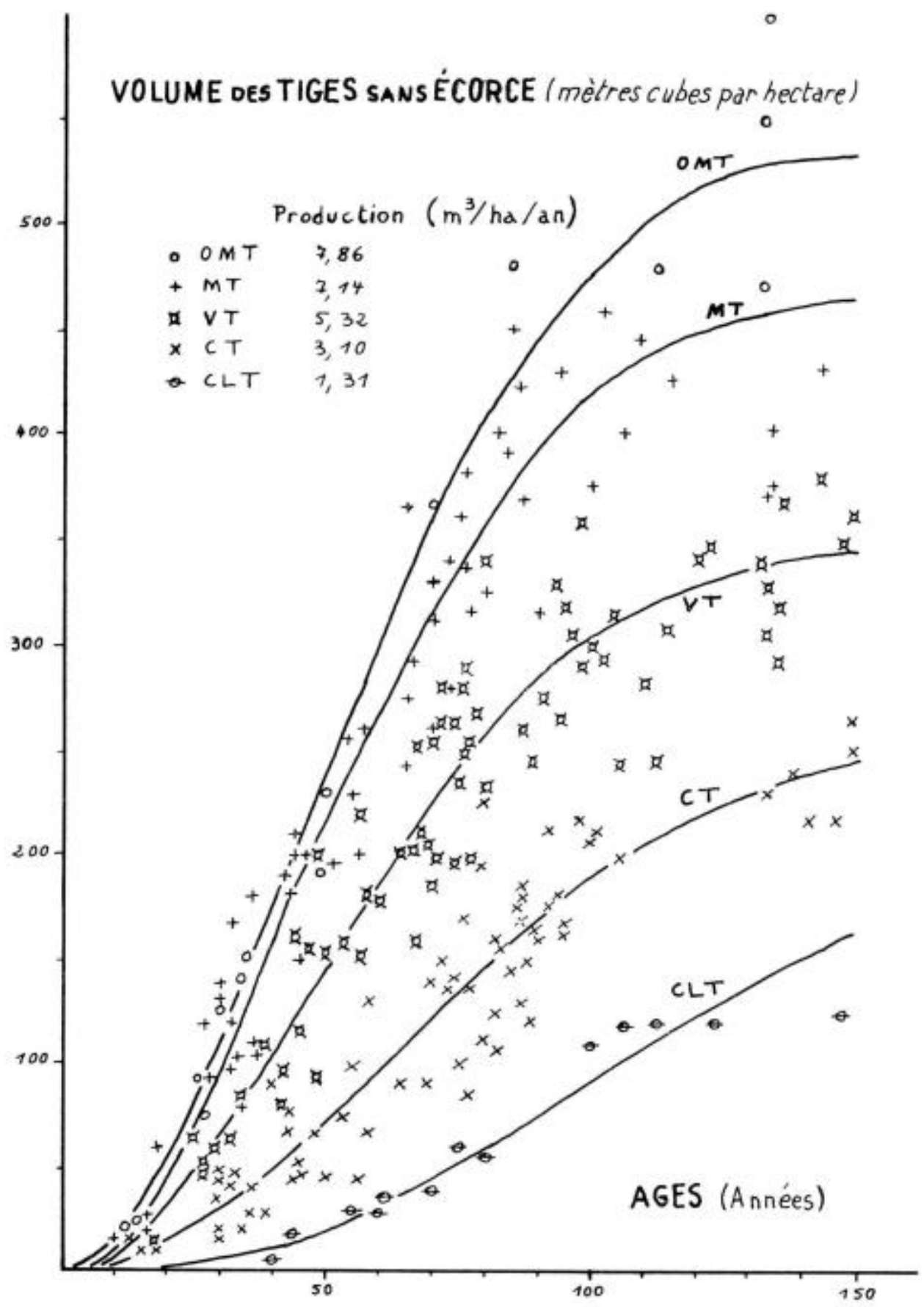

FIG. 1. - Exemple d'utilisasion des types forestiers de Cajander Productwité des forifs finlandaises :

$O M T=t y p e$ à Oxalis et Myrtillus;

$M T=$ type a Myrtillus:

$V T$ - type à Vaccinium:

$C T$ - type à Calluna:

CLT - type à Cladina (d'après llvessalo, 1920) 
Quoi qu'il en soit, étant admis que la productivité d'une station est limitée par les variables qui y sont les plus défavorables - c'est la loi du minimum —, leur recherche et leur utilisation comme critères de production peut être fort intéressante (MitcherLiCH, 1950, Colle, 1948, Tamm et Wadman, 1945, Oswald, 1969, Decourt et Le Tacon, 1970). Soulignons encore que, beaucoup plus que pour les critères purement floristiques, l'utilisation de ces critẻres écologiques est très strictement limitée aux zones oủ toutes les autres variables écologiques restent sensiblement constantes.

\section{Critères phyto-écologiques}

Les études où critères floristiques et critères écologiques sont utilisés conjointement sont plus rares, du fait de leur caractère pluridisciplinaire. Le travail de DAGNELIE (1956), dans les hêtraies de l'Ardenne belge, est à cet égard une étape importante. Les critères utilisées ne sont pourtant pas des critères phyto-écologiques au sens strict. L'auteur étudie en fait successivernent la corrélation flore-production, puis la corrélation facteurs écologiques-production; il conclut d'ailleurs à la nette supériorité des critères floristiques.

D'autres études, menées dans des forêts françaises, ont également eu pour but de définir les types de stations à un triple point de vue : floristique, écologique (et principalement édaphique) et productif. Il s'agit essentiellement des travaux de Duchaufour et MiLlischer (1954) en forêt de Bussang (Vosges), puis de Duchaufour, Parde, Jacamon et Debazac (1958) en forêt du Ban d'Étival (Vosges) (voir fig. 2 et tableau 1). Bien que les études phytosociologique et pédologique aient été menées séparément, elles ont fait l'objet d'une synthèse écologique du plus haut intérêt. D'autre part, l'approche phytosociologique a été grandement facilitée par l'utilisation des groupes écologiques, c'est-à-dire de groupes de plantes déjà établis, ayant des exigences écologiques semblables et connues; dans ce cas, on peut alors parler de véritables critères phyto-écologiques.

La possibilité d'utiliser d'embléc de tels groupes écologiques est une aide très appréciable pour les études de massifs forestiers; celles-ci peuvent être relativement " légères \# et reposer sur un nombre restreint de relevés « bien choisis "; les données ainsi recueillies se prêtent donc aisément à être traitées par des moyens traditionnels simples.

Une remarque importante doit cependant être formulée. Notre " arsenal » de groupes écologiques ainsi directement utilisables est incomplet, et il n'est valable que pour certaines régions privilégiées - comme le Nord-Est de la France —, oủ ils sont le fruit d'une longue expérience de forestiers biologistes tels que Guinier, Rol, Duchaufour, JaCamon, Étant donné l'intérêt manifeste de ces critères phyto-écologiques pour l'identification et la cartographie des stations, et la prévision de leur productivité, en quantité comme en qualité, il nous est apparu urgent de généraliser le concept de groupe écologique à une beaucoup plus vaste échelle.

\section{L'ÉLABORATION DES GROUPES ÉCOLOGIQUES}

Un des premiers objectifs à atteindre était de mettre au point un ensemble de méthodes aptes à étalonner rapidement, dans une région forestière mal connue, le précieux instrument de mesure que corstitue la végétation naturelle, c'est-à-dire à en élaborer les groupes écologiques. 
S

7................ I - . .

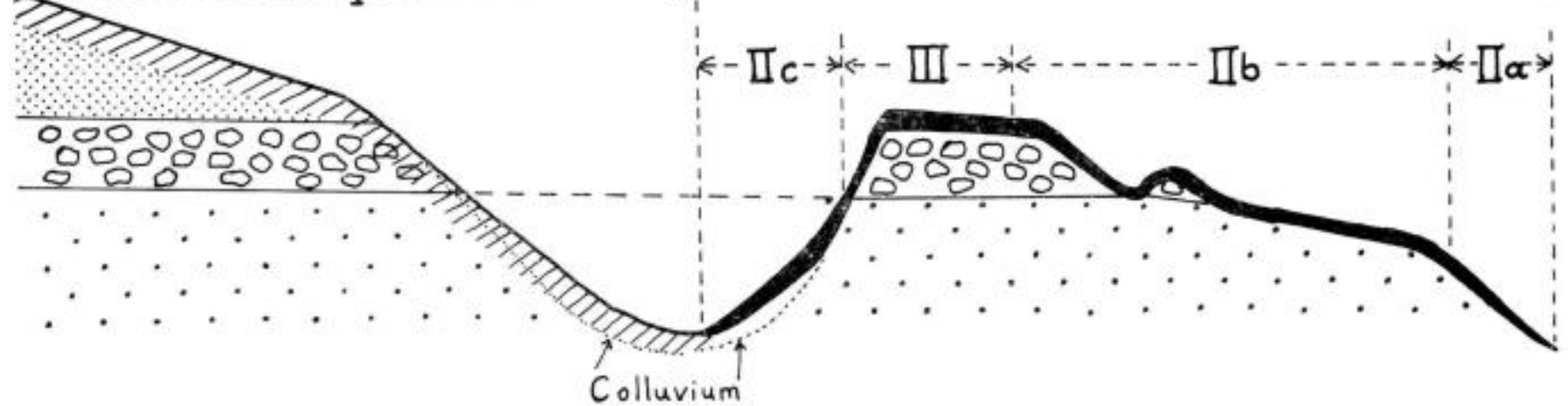

Types d'humus

D2 Mull

Humus brut \$rès intermédiaire

00 conglomérat

$\therefore$ grès vosgien

FiG. 2. - Coupe schématique montrant la répartition dex types de stations en forèt du Ban d'Etival (88) (d'après Duchaufour et al., 1958) 
TABLEAU 1

Ecologie et productivite des divers types de stations en forét du Ban d'Etival (88) (d'après Duchaufour et al., 1958)

\begin{tabular}{|c|c|c|c|c|}
\hline $\begin{array}{l}\text { Numéro } \\
\text { du type }\end{array}$ & $\begin{array}{l}\text { Peuplement et } \\
\text { Productivité } \\
\left(\mathrm{m}^{\mathrm{a}} / \mathrm{ha} / \mathrm{an}\right)\end{array}$ & Végćtation & Stations & Sol \\
\hline I & $\begin{array}{l}\text { Sapin } \\
\text { Hêtre }\end{array}$ & $\begin{array}{l}\text { Festuca silvatica } \\
\text { Polystichum spinulosum } \\
\text { Rubus glandulosus } \\
\text { Oxalis acetosella }\end{array}$ & $\begin{array}{l}\text { 1. Grés intermédiaire } \\
\text { assez riche en argile } \\
\text { 2. Sols colluviaux drainés } \\
\text { sur versants frais }\end{array}$ & $\begin{array}{l}\text { Sol brun a mull } \\
\text { acide. }\end{array}$ \\
\hline II & $\begin{array}{c}6,5 \mathrm{~m}^{3} \\
\text { Sapin } \\
4,5 \mathrm{~m}^{3}\end{array}$ & 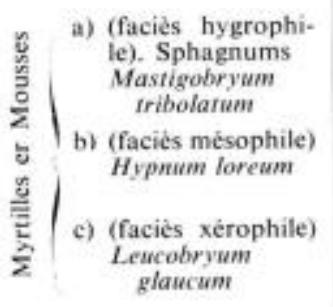 & $\begin{array}{l}\begin{array}{l}\text { Sols colluviaux mal } \\
\text { drainés }\end{array} \\
\text { Grés vosgien, plateaux et } \\
\text { pente; exposition de tran- } \\
\text { sition } \\
\text { Pentes fortes aux exposi- } \\
\text { tions chaudes à colluvium } \\
\text { grossier. }\end{array}$ & $\begin{array}{l}\text { Sol de la série } \\
\text { podzolique a } \\
\text { moder ou mor } \\
\text { (plus ou moins } \\
\text { évolué) }\end{array}$ \\
\hline III & 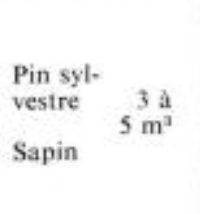 & $\begin{array}{l}\text { Calluma valgaris } \\
\text { Hypnum schreberi }\end{array}$ & $\begin{array}{l}\text { 1. Dalle de conglomérat } \\
\text { (taches de Molinia coe- } \\
\text { rulea et de Sphagnums) } \\
\text { 2. Sols colluviaux de } \\
\text { pentes exposees au Sud }\end{array}$ & $\begin{array}{l}\text { Sol d'humus brut } \\
\text { sur dalle. } \\
\text { Podzol humo- } \\
\text { ferrugineux à } \\
\text { alios et a mor } \\
\text { epais }\end{array}$ \\
\hline
\end{tabular}

Or le développement considérable, depuis quelques années de puissants outils de tri et de calcul, nous a permis d'envisager la poursuite de cet objectif selon des démarches nouvelles. En particulier, le nombre de données élémentaires recueillies sur le terrain n'est plus un facteur limitant, ce qui peut retentir profondément sur la qualité de l'échantillonnage des points d'observation (appelés " relevés »).

Par essence même, le groupe écologique contient deux sortes d'information : sa composition floristique d'une part, sa signification écologique d'autre part. Au cours de son élaboration, l'une ou l'autre de ces informations peut précéder l'autre :

- ou bien on commence par rassembler les espèces dont on constate les affinités sociologiques, pour définir ensuite écologiquement les milieux dans lesquels les groupes ainsi formés sont les mieux représentés,

- ou bien on commence par rapprocher les relevés dont les caractéristiques écologiques paraissent ou ont été reconnues semblables, et l'on observe les espèces qui sont les plus fidèles aux types de milieux ainsi définis.

Dans l'absolu, si les techniques d'analyse du milieu écologique et celles relatives à la végétation étaient parfaitement au point, il devrait y avoir identité entre les résultats obtenus par l'une ou l'autre démarche. La faveur qu'ont pu connaître l'une ou l'autre nous semble 
en fait avoir été conditionnée par le degré de perfectionnement des outils - et, secondairement, des méthodes - mis à la disposition du chercheur.

Bien que ne s'appliquant pas à la notion de groupe écologique, il convient de souligner que la première démarche est celle de la phytosociologie « sigmatiste » ${ }^{1}$ ) la plus orthodoxe. Mais, trop souvent, seule la première phase de l'approche a été menée, à savoir l'élaboration des associations végétales; leur caractérisation écologique était ensuite laissée dans l'ombre ou faite succinctement.

Le développement du matériel mécanographique (trieuses, tabulatrices) a permis de donner une dimension nouvelle aux études phyto-écologiques. Celles-ci ont pu porter sur de trẻs nombreux relevés, répartis selon un échantillonnage beaucoup plus objectif que par le passé, et comportant, en plus des listes floristiques classiques, des observations écologiques en nombre pratiquement non limité. La méthode la mieux formalisée est celle des groupes écologiques statistiques de Gounor (1959).

La méthode Gounor procède plutôt de la seconde démarche énoncée ci-dessus. A partir des relevés floristico-écologiques effectués, des histogrammes sont construits qui traduisent le nombre d'apparitions de chaque plante pour chacune des classes de chacune des variables écologiques mesurées. Les espèces dont les histogrammes ont un "profil » semblable pour l'ensemble de ces variables sont alors réunies en groupes écologiques provisoires, dont on teste, a posteriori, la réalité sociologique.

Il s'agit là de l'une des deux méthodes qui ont été utilisées il y a quelques années pour notre première étude de massif forestier, au début des activités du Laboratoire de Botanique forestière (AuSSENAC, BECKER, 1968). L'autre méthode mise en œuvre alors, grâce à l'aide de la station de Biométrie du C.N.R.F., et notamment de MuLuER, nécessite l'utilisation d'un ordinateur. Du fait des caractéristiques de l'engin disponible alors, la technique d'analyse multivariable envisagée (analyse des composantes principales) ne pouvait être appliquée aux variables floristiques, trop nombreuses. C'est donc également la seconde démarche qui a été suivie : analyse purement écologique, suivie d'une classification automatique des espèces selon leur réponse aux variables écologiques (voir fig. 3).

II faut être conscient du fait que les résultats obtenus par une quelconque des méthodes basée sur la seconde démarche n'ont de valeur certaine que dans la mesure où la partie écologique de l'étude a été correctement menée. En particulier, l'oubli ou la mesure incorrecte d'un facteur important du milieu, se traduit automatiquement par de sérieuses difficultés au moment de la classification des espèces ; avec la méthode faisant appel à l'analyse multivariable, cela se traduit par l'élaboration de groupes d'espèces peu convaincants sur le plan sociologique; avec la méthode Gounot, les premiers groupes provisoires formés doivent être longuement retouchés, par tâtonnements, avant d'aboutir à des groupes résistant aux tests imposés, dont la signification écologique reste de toutes façons imprécise.

Ce fut le cas, par exemple, au cours de la phase d'inventaire phyto-écologique lors de l'étude de la forêt de Charmes : un des facteurs décisifs de l'écologie était le degré d'hydromorphie des sols, et, pour le traduire, nous avions essentiellement misé sur la profondeur d'apparition de l'horizon Bg, plancher supposé des nappes temporaires; or, fait fort intéres-

1. Du sigle S.I.G.M.A. : Station internationale de Géobotanique méditerranéenne et alpine, Montpellier. 


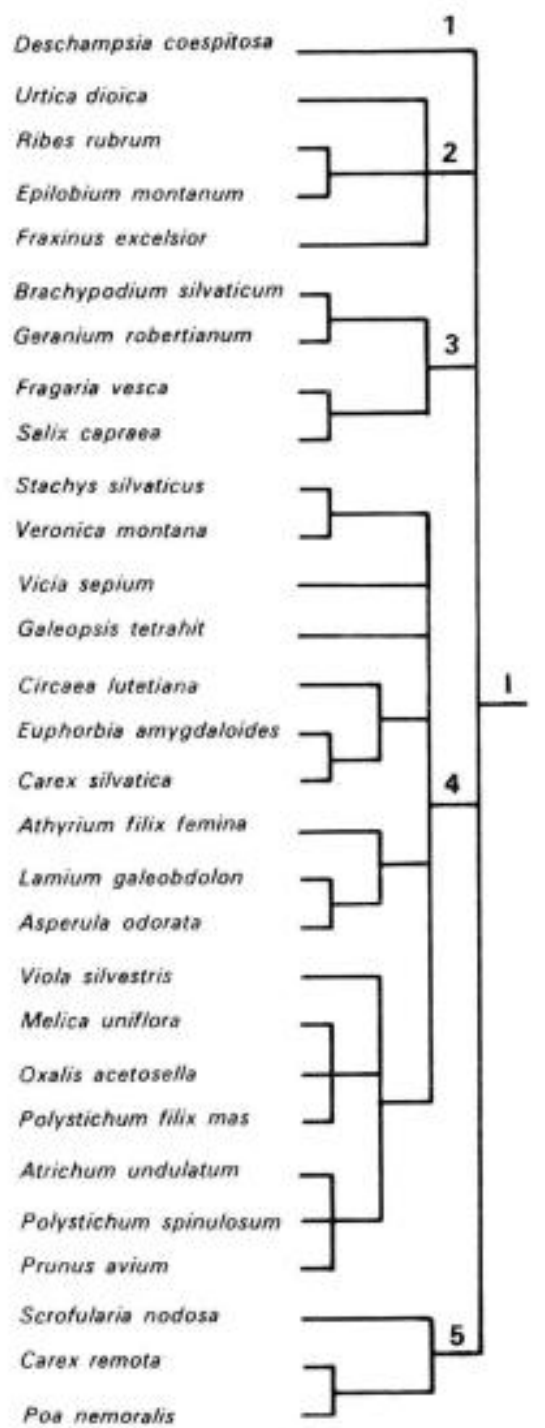

Lysimachia nemorum

Stellaris holostea

Betula verrucose

Carex pahescens

Moehringia trinervia

Juncus sp.

Quercus sp.

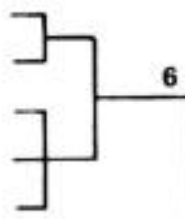

Carpinus betufus (arbustif)

Hedera helv

Minum effusum

Anemone nemorosa

Acer psecroloplatamis

Agrostis $s p$

Epilobium angustiforium

Rubers idaeus

Sambucus nigra

Calamagrostis epigeros

Lezula forstari

Sarothamness scoparius

Ruscus aculeatus

Endymion non scriptum

Lonicera periclymenum

Rubus 50

II

Mex aquifolium

Pairgonatum meitiflorum

Polytrichum formosum

Carex pilulifera

Holcus mallis

Pteridium aquilinum

Sorbus aucuparia

Convallaria majalis

Deschampsia /lexuosa
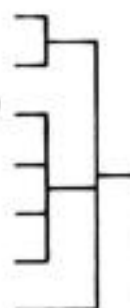

6
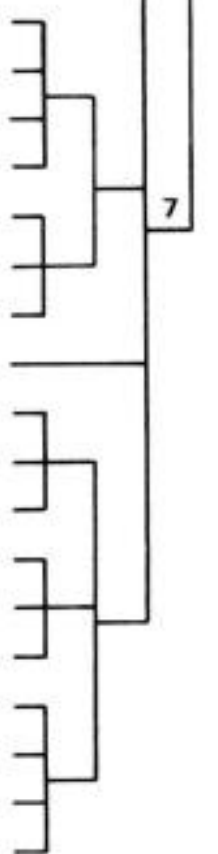

$\square$

FIG. 3. - Exemple de classification automatique des espèces selon leur réponse aux variables feologiques (Fonêt de Villers-Corterérs, Becker 1969) 
sant d'ailleurs, cet espoir s'est avêré en grande partie infondé, du fait du caractère relique de cet horizon - la suite de l'étude devait en apporter la preuve (voir fig. 4).

Une attention toute particulière fut donc apportée au choix des variables écologiques à mesurer pour l'étude suivante menée en forêt de Villers-Cotterêts (Aisne) à propos des problèmes soulevés par le Hêtre sur substrat siliceux (BECKER, 1969). D'autre part, grâce au perfectionnement des moyens de calcul et des programmes disponibles, une première tentative a été faite de l'utilisation de la première démarche, parallẻlement à la mise en cuvre de la seconde. Bien que, pour des raisons techniques, l'analyse purement floristique toujours basée sur l'analyse des composantes principales - $n$ 'ait pu être faite que sur une partie des espèces inventoriées (la moitié environ), les groupes obtenus coĩncidèrent de façon remarquable avec ceux issus de l'autre approche; les deux démarches confirmaient donc mutuellement leur valeur.

Peu de temps après, n'étant pratiquement pius limité par le nombre d'espèces, Timbal (1968) s'engageait dans la voie purement floristique, à l'occasion de son étude en forêt de Sainte-Hélène (Vosges), et parvenait, de façon très satisfaisante, à mettre en évidence groupes sociologiques et associations végétales. L'essentiel de la partie écologique de l'étude de ce massif avait - rappelons-le — déjà été traitée par ailleurs (LE TACON, 1966).

Dans son étude des forêts sur Rhétien, PICARD (1970) entreprenait une ultime comparaison des deux démarches possibles énoncées plus haut. Il concluait à la plus grande sûreté des groupes obtenus par la voie strictement floristique.

L'ensemble de ces résultats, obtenus par étapes successives, nous permettent aujourd'hui de préconiser une approche méthodologique qui, si elle devait être adoptée par une majorité des phyto-écologistes, marquerait incontestablement pour eux une étape importante. D'autant plus importante qu'elle devrait permettre à des phytosociologues actuellement très divisés de retrouver enfin une unité de vue très souhaitable. Soulignons encore combien fut décisive, dans cette progression, l'aide de la Station de Biométrie du C.N.R.F., et notamment de Millier.

Cette « nouvelle " approche n'est d'ailleurs que le retour, avec de nouveaux outils, à la première démarche, dont certains d'ailleurs, ne s'étaient jamais départis. Ainsi que l'affirme avec force Guinochet (1966) : «Des considérations précédentes... sur les relations réciproques entre végétation et milieu, il résulte que, contrairement à une idée solidement, mais fâcheusement ancrée dans la majorité des esprits, celui-ci correspond à un concept très relatif. Exprimons-nous franchement : s'il $n^{\prime} y$ avait pas de plantes, nous n'aurions pas à nous interroger sur leurs conditions d'existence; autrement dit, la classiquement sacrosainte notion de milieu ne nous viendrait même pas à l'esprit. Le milieu, conçu comme l'ensemble des conditions environnantes, ne peut donc être défini que par rapport à un objet d'étude - association végétale, population, espèce, individu, etc. ".

Les études phyto-écologiques engagées actuellement par le Laboratoire de Botanique forestière sont done conçues en deux étapes complémentaires. Les relevés effectués au cours de la première étape sont uniquement floristiques, selon la méthode classique de BrauNBlanquet. Allégés de toute la partie écologique, qui prenait beaucoup de temps, ils peuvent être très nombreux. Leur analyse conduit à dègager un certain nombre de groupes "sociologiques ( ou " coenologiques $»)$ dont il reste, au cours d'une seconde étape à dégager, ou à préciser, la signification écologique exacte. Un deuxième échantillonnage beaucoup plus 

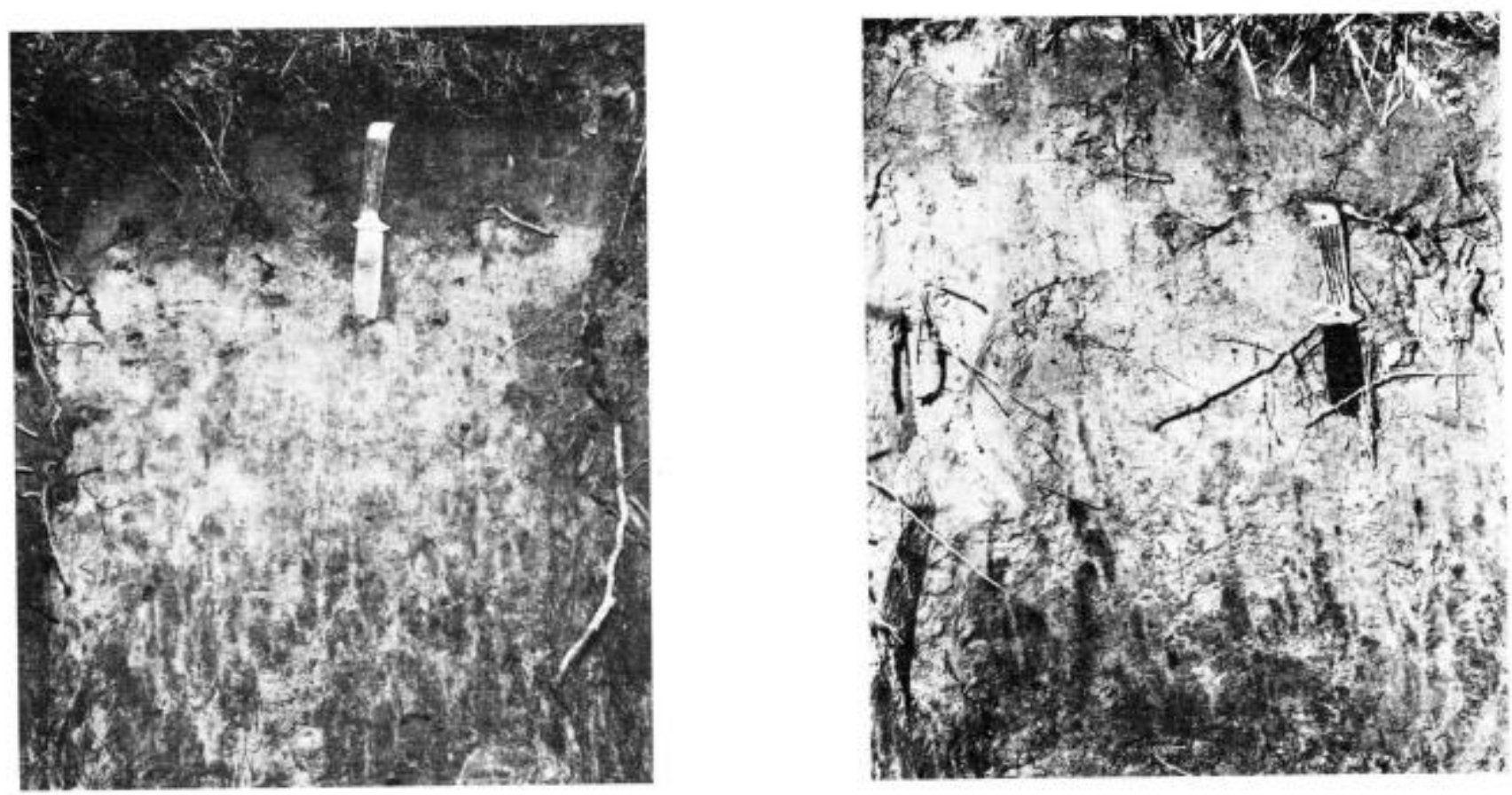

Fio. 4. - Certains critères peddologiques (ici la profondeur de Thorizon Bg)

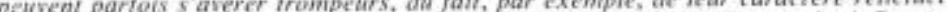
la végétation, entre autres, permut d'éveiller les soupsons (A : station hydromorphe à Carex brizoides; B- station saine à Poa chaixi), (d'après Becker, 1971 ) 
restreint de relevés - ceux qui caractérisent le mieux les groupes sociologiques de référence - est établi. Ces relevés peuvent alors faire l'objet d'une analyse écologique plus ou moins poussée; la comparaison des résultats obtenus doit alors permettre de conférer aux groupes sociologiques leur label de " groupes écologiques"

Les premiers résultats obtenus actuellement par Timbal dans son étude des Hêtraies du Nord-Est de la France sont extrêmement encourageants. Prolongement de la méthode d'analyse des composantes principales, la méthode d'analyse factorielle des correspondances qui a été utilisée semble très séduisante. Signalons en outre que cette étude est un des sousensembles d'un projet de recherches multidisciplinaire beaucoup plus vaste, et que la seconde étape dont nous venons de parler sera, de façon très heurcuse, un des "sous-produits" fort intéressant pour nous de l'activité des autres spécialistes. Étant donnée l'économie d' " énergie " qui en découle, cette façon de procéder est évidemment à rechercher dans toute la mesure du possible.

\section{PREMIÉRE APPROCHE DE L'APPRÉCIATION DE LA PRODUCTION}

A quel moment préconiser l'appréciation de la production, en quantité et en qualité, dans les stations caractérisées par les groupes écologiques ainsi formés, et comment? En principe, il semble logique de reporter cet aspect en deuxième phase, en le confiant aux spécialistes qualifiés. C'est ce qui a d'ailleurs été fait pour l'étude en forêt de Villers-Cotterêts (aspect quantitatif seul) déjà citée, et ce qui est prévu pour celle en cours des Hêtraies de I'Est.

Il nous semble en fait possible de favoriser un allègement des opérations correspondantes, de toutes façons nécessaires puisque seules quantitatives, ou de leur donner une portée plus grande, en esquissant qualitativement cet aspect au cours de la première phase. II se trouve que notre travail de terrain, pour des raisons techniques, se fait en général à deux opérateurs. Or un seul suffit pour le relevé floristique; l'autre se trouve donc disponible pour une autre mission, dont la durée doit correspondre sensiblement à celle du relevé floristique. Celle que nous lui confions consiste essentiellement en la mesure de la hauteur d'un ou deux des arbres dominants les plus représentatif́s de la station, et de leur circonférence à $1,30 \mathrm{~m}$; une carotte est également prélevée à la tarière de Pressler. Certes, ces mesures n'ont guère de valeur pour un relevé pris séparément; par contre, il nous semble du plus haut intérêt de considérer toutes celles effectuées dans les relevés caractérisés par un groupe sociologique donné, et d'obtenir ainsi, avec une précision suffisante, un indice en liaison étroite avec la productivité du type de station correspondant (voir tableau 2).

De la même façon, nous croyons en l'intérêt de la mesure, sur un ou deux arbres seulement, du couple de torsion nécessaire à l'enfoncement de la tariẻre de Pressler, selon la technique mise au point par Polge et Keller (1970); selon ces auteurs, celle-ci est en liaison très étroite avec un certain nombre des caractéristiques technologiques du bois. Là encore, naturellement, il est indispensable que le spécialiste " ès qualités du bois " intervienne au cours de la deuxième phase pour étudier de façon plus complète la question, sur un nombre minime de relevés qui deviendront autant de points de référence pour toutes les stations à même composition floristique. 
TABLEAU 2

Hautesur des néserves d'äge supêrieur à 140 ans en Forêt communale de Charmes (d'aprés BeCKer, 1971)

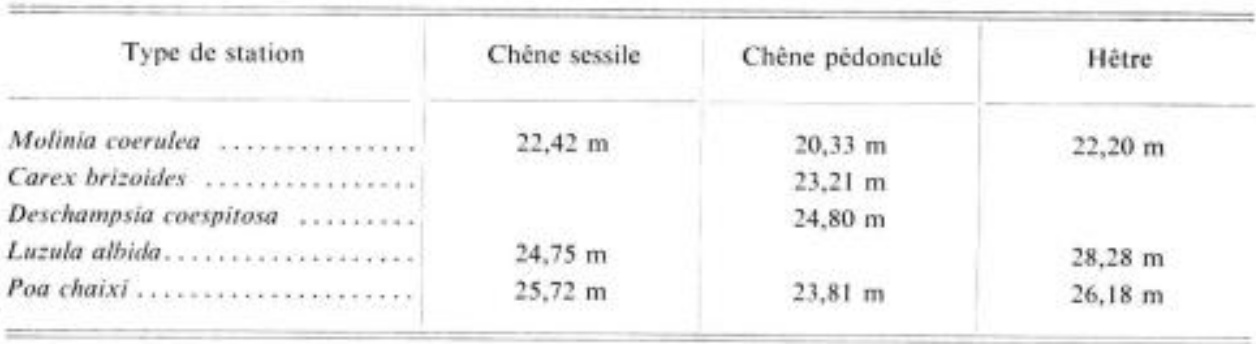

\section{CONCLUSION}

Nous pensons donc être parvenus à l'heure où la phytosociologic forestière - si on lui en donne les moyens - peut devenir « opérationnelle». Nous sommes intimement persuadés, d'ailleurs, qu'il en résulterait une meilleure économie des moyens globaux mis à la disposition des recherches forestières. En effet, l'inventaire floristique d'un territoire est une operation beaucoup plus rapide, et surtout bien moins onéreuse que tous les autres types de prospections; et il semble souhaitable d'y avoir recours chaque fois que cette démarche est possible.

En particulier, l'élaboration de la stratégie des projets multidisciplinaires peut en être facilitée et gagner grandement en " fiabilité ». II serait en effet regrettable, à ces occasions, soit d'ignorer le phytosociologue, soit de ne faire appel à lui que tardivement, une fois les dispositifs choisis et les protocoles définis, partie, peut-être, pour lui faire plaisir, partie par acquit de conscience, un peu de la même façon que, dans les généralités d'une étude, on rappelle les données géologiques et macroclimatiques de la région.

Cet aspect n'est bien sûr pas dénué d'intérêt. Le phytosociologue peut pourtant apporter beaucoup plus aux autres spécialistes, mais à condition d'intervenir avant eux. Le choix de l'emplacement des dispositifs est une phase capitale et parfois trop peu raisonnée; or la phytosociologie peut faciliter ce choix avec les meilleures garanties d'objectivité. D'autre part, grâce à la connaissance qu'il possède déjà de la signification écologique de la flore, il peut également orienter utilement les autres spécialistes dans le choix - capital — des variables écologiques à étudier.

\section{SUMMARY}

\section{ECOPHYTOSOCIOLOGY AND LIGNEOUS PRODUCTION}

The definition of isopotential sites (according to quality and quantity production) can be considered with various indices. Beyond pure dendrometric, or pure ecological indices - studied in other communications - spontaneous vegetation can be often a very convenient tool. There are pure floristical indices, which are used since a long time in boreal forests (CAJANDER, 1926). As 
they seemed ill-adapted to temperate forests complexity, one had to wait for the idea of \& ecological group ", and mainly the studies of DUChaUfour, to see used true phyto-ecological indices. These ecological groups (groups of plants characterized by similar ecological needs) are useful for defining and mapping of forest sites. They can be elaborated by means of more or less complex methods : traditional method of phylosociological tables; statistical ecological groups (Gounor); principal components analysis; factor analysis... The ecological interpretation can, according to the cases, follow or precede the floristical analysis. The valuation of site productivity can be considered in two stages : qualitative during the preliminary phytozcological study; then quantitative during the more detailed ecological study of the so determinated sites.

\section{RÉFÉRENCES BIBLIOGRAPHIQUES}

Aussznac G., BeCKer M.. 1968, - Ecologie d'un massif sur sols hydromorphes : la forêt de Charmes (Vosges). Contribution à la mise au point d'une méthode d'étude dynamique du milieu forestier. Ann. Sci. forest.. 25, (4), 291-332.

BARTOL Ch., 1966. - Études écologiques sur les associations forestières de la Haute-Maurienne. Ann. Sci. forest., 33, (3), 3-321.

Beckfr M., 1969, - Le Hêtre (Fagus silrasica L.t et ses problèmes en forèt de Villers-Cotterèts (Aisne). Contribution à la mise au point d'une méthode dynamique d'étude écologique du milieu forestier. Ann, Sci, forest., 26, (2), 141-182.

Becker M., 1970. - Écologie et phytosociologie : sciences de base en recherches forestières. Bull. Acad. Soc. lorr. Sci, 9, (1), 11-15.

BECKER M., 1971. - Elude des relations sol-vigération, en conditions d hydromorphie, dans une forét de la plaine lorraine. Thèse État. Nancy 1, 225 .

CaJANDER A, K., 1926. - The theory of forest types. Acta forest. fenn. 31, 108.

CAJAnder A. K., 1943. - Nature et importance des types de foréts. Intersy/va, 3, 169-209.

Colle T. S., 1938. - Classification of forest sites with special reference to ground vegetation, fourn. For., 36. $1062-1066$

Dagnelie P., 1956-1957. - Recherche sur la productivité des hêtraies d'Ardenne en relation avec les types phytosociologiques et les facteurs écologiques. Bull. Inst. agron. Stat, Rech. Gemblowx, 24, 249-284 et $369-410 ; 25,44-94$.

Dagnelue P., 1957. - Quelques donnèes nouvelles sur la productivité des hètraies d'Ardenne. Bull. Sor. roy. forest, Belg., 12, 515-534.

Dagnelie. P., Hunerty J. P.. Notrfalise A., 1960, Recherches sur la productivité des hètraies des macignos et des mames du Bas-Luxembourg. Bull. Inst. agron. Stat. Rech. Gembloux, 28, 1, 18-32.

Dagver.te P., 1950. - Contribation à l'étuje des cammunauté; végétales par l'analyse factorielle. Bulf. Serv. Carte phytogéogr, B, 5, 1-195.

Decourt N.. LE TacoN F., 1970. - L'Epicéa commun (Picea excelsa) sur les plateaux calcaires de l'Est de la France. Essai de prévision de la production à l'aide de déterminations pédologiques simples. Ann. Sci. forest., 27, (3), 255-286.

Duchaufour Ph., 1948. - Recherches écologiques sur la chēnaie atlantique française. Ann. E.N.E.F. 332.

Duchaufour Ph. Millscher H., 1954. - Êtude des types de végétation dans une sapinière vosgienne. Rev, forest. franc., 3, 160-178.

Duchaufour Ph., 1957. - Tableasx descriptifs et analytiques des sols. École nationale des Eaux ef Foríts, Nancy, 85.

Duchaufour Ph., Parden J., Jacamos M., Debuac E. F., 1958. - Un exemple d'utilisation pratique de la cartographie des station; : la forżt du Ban d'Etival (Vosges). Rev. forest. franc., 10, 597-630.

Duchaupour $\mathrm{Ph}$., 1960. - Stations, type d'humus et groupements écologiques. Rev. forest. franc., 7, 484494.

Duchaufour Ph., Bonnzau M., Dfanzac E. F., Parde J., 1961. - Types de forêts et amènagement : la forêt de la Contrôlerie en Argonne. Annales Ecole nasionale des Eaux et Forírs, 18, (1), 1-44.

Duyigneaud P., Denayer DE SMet S., 1970. - Phytogéochimie des groupes écosociologiques forestiers de Haute Belgique. Erol. Plant., 5, 1-32.

Ellevizrg H. et al., 1967. - Vegetations - und bodenkundliche Methoden der forstlichen Standortskar tierung. Veroffentl, geobotan. Inst. E.T.H.. 39, 296.

Garbaye J., Leroy Ph., Le Tacon F., Levy G., 1970. - Réflexions sur une mèthode d'études des relations entre facteurs écologiques et caractéristiques des peuplements. Ann. Sci. forest., 27, (3), 303-321.

Gopron M. et al., 1968. - Code pour le relevé méthodique de la végétation el đu milieu. C.N.R.S., Paris, 292. 
Gounot M., 1959. - L'exploitation mécanographique des relevés pour la recherche des groupes écologiques. Bull. Serv. Carle phytogéogr., B, 4, 147-177.

Gounor M., 1969, - Méthodes d'étude quantitative de la végétation. Masson, Paris, 314.

Guinier Ph., 1959. - Le milieu édaphique et la composition floristique des foréts. Soc, bot. Fr., I er colloque, Paris, 114-120.

Guinocher M., 1966. - L'écologie végétale : quelques remarques sur ses fondements et ses objectifs. Mises á jour, 1, 387-402.

It.vessalo Y., 1920. - Ertragstafeln für die Kiefern —. Fichten — und Birkenbestände in der Südhälfte von Finnland. Acra forest. Jenn., 15.

Jacamon M., Mormche A., 1958. - Une étude pédologique et botanique en liaison avec celle des types de peuplements (Forêt domaniale des Trois-Bois). Rev, forest. franc., 2, $81-94$.

Jacques-Félux H., 1959, - Rapport du sol et de la végétation. Introduction. Soc. bot. Fr., 1er Colloque, Paris, $1-10$.

Lemíz G., 1954. - Pédologie et Phytosociologie. VIII* Congr. Inst. Bot., Paris, rapport comm. sect. 7 et 8, 33-38.

Мтsснекцси G., 1950. - Die Bedeutung der Wuchsgebiet für das Bestandeswachstum von Buche, Eiche, Erle und Birke. Forstwiss. Centralbl, 69, 184-211.

Oswal. H., 1969. - Conditions forestières et potentialité de l'Épicéa en Haute Ardèche. Ann. Sci. forext., 26, (2), 183-224.

PiCard J. F., 1970. - Les foréts sur Rhétien dans le dèpartement des Vosges. Nouvelle contribution à la mise au point d'une méthode dynamique d'étude phyto-écologique du milieu forestier. Thése 3 eycle, Nancy, $74 \mathrm{p}$.

Polge H., Kellek R., 1970, - Première appréciation de la qualité du bois en forét par utilisation d'un torsiometre, Ann. Sci. forest., 27, (2), 197-223.

Ruaner K., 1923. - Die Bedeutung der Waldtypen für den deutschen Wald. Silva.

RUaver K., 1934. - Der gegenwärtige Stand der Waldtypenfrage. Zeitschrifi fïr Weltforstwirtschaft, März, Neudamm.

Scheffer L., 1944. - Les types de foréts de CauAnder. Rev. Eaux For., 82, 245-263.

TAmm O., Wadman E., 1945. - Sur les facteurs stationnels et leur corrélation avec les conditions forestières dans le district de Hamra (texte suédois). Svenska Skogs. Tidskr., Bihang, 2, 79.

Timbal J., 1968. - Contribution à l'étude des associations forestières de la forét de Sainte-Helène (Vosges). Thèse, 3e cycle, Orsay, 104.

Tıмва J., 1970. - Difficultés rencontrées par la phytosociologie dans les milieux forestiers, Bull. Acad. Soe, lorr. Sci., 9, (1), 199-210.

VAL.tée G., 1967. - Utilisation des relations entre la végétation du sous-bois et la productivité des stations pour la classification des foréts. Thèse, Doct. Ing., 2e sujet, Nancy.

\section{DISCUSSION ET INTERVENTIONS}

M. Pourtet. - A propos des « types forestiers » de CANANDER, je signale qu'au début du 19" siècle, déjà, un récit de voyage d'exploration botanique en Amérique du Nord de Mıchaux fils, rapporte que, dans la région des Appalaches, la valeur-argent des terres attribuées aux colons était fixée en fonction de l'essence principale qui y croissait naturellement. Ainsi le prix le plus bas correspondait-il aux stations à Pinus banksiana, le prix moyen à celles à Quercus rubra, et le plus élevé à celles à Liriodendron tulipifera.

M. Duchaufour. - Une phrase de M. Becker m'a quelque peu inquiété : c'est celle qui concerne le retour à une méthode purement floristique d'étude de la végétation (précédant bien entendu l'étude écologique). Cette méthode me paraît représenter un réel danger de perte de temps; on peut se demander, en effet, quel sera le critère du choix des stations sur lesquelles seront pratiqués les relevés. Selon nous, un tel choix ne peut être livré au hasard et doit résulter d'une sélection très fine, basée sur l'expérience de l'observateur, acquise antérieurement. Nous donnerons deux exemples de tels choix, permettant de comparer des stations ne différant entre elles que par un petit nombre de facteurs écologiques : 1) Méthode 
utilisée par BARTOL en Maurienne : étude des variations des groupes écologiques observés en circulant le long d'une courbe de niveau à différentes expositions, ou au contraire le long d'une ligne de plus grande pente, permettant de dégager l'influence de l'altitude. 2) Exemple en plaine (forêt de la Contrôlerie en Argonne) : la base écologique des relevés était le type d'humus, qui intègre tout un ensemble facteurs, humidité, aération, $\mathrm{pH}$, etc.

M. BECKER. - La démarche que nous proposons (floristique seule dans un premier temps, écologique dans un deuxième temps), ne s'applique pas à l'étude des situations forestières proprement dites, mais à l'étalonnage préalable de la végétation dans une région donnée, c'est-à-dire à la formation des groupes écologiques. Bien entendu, une fois cette phase préalable menée à bien, les études de massifs qui sont alors entreprises peuvent, et doivent l'être selon les principes énoncés par M. Duchaufour : utiliser les groupes écologiques ainsi formés, mais aussi un certain nombre de critères écologiques plus ou moins détaillés, qui orientent les prospections ou qui viennent compléter l'information fournie par la vêgétation.

M. Godron. - II est en fait possible de concilier les exigences probabilistes (relevés faits « au hasard ») ct l'utilisation des connaissances déjà acquises, par un échantillonnage « stratifié »; c'est maintenant une méthode couramment utilisée.

Par ailleurs, nous avons suivi, au C.E.P.E., une evolution assez proche de celle que nous a présentée $\mathrm{M}$. BECKER, bien que nous maintenions, sur le terrain, la collecte de données écologiques. C'est surtout dans la succession des calculs que nous plaçons les groupes conologiques avant les groupes écologiques; ceux-ci sont d'ailleur3 souvent formés pour répondre à des demandes précises des utilisateurs (forestiers, agronomzs, etc.). Comme cela vient d'être dit, le rapprochement des points de vae des diverses "écoles " phytosociologiques est un des événements heureux des dernières années. Il permat d'ailleurs de penser que la mise en commun des observations phyto-écologiques dzvient possible, pour constituer des «écothéques" accessibles et utiles à tous.

M. Sauvage. - Il est très important de bien rappeler - comme l'a du reste fait M. BECKER - que l'utilisation de la flore pour caractériser un milieu n'est sûre que dans des limites géographiques étroites. Les caractéristiques fioristiques ne sont pas exportables.

Je pense par ailleurs que, dans un pays dont on ne connait pas la flore (et sa valeur indicatrice), il faut absolument mener l'analyse écologique de pair avec l'analyse foristique.

M. Gounot. - En fait, le choix de la méthode de travail dépend essentiellement de l'échelle du problème. Il me parait également très dout zux qu'il soit possible de débrouiller les groupes écologiques d'une rẻgion ètendue et écologiquement complexə d'une manière purement floristique.

M. BeCKer. - Nous sommes parfaitement d'accord sur ces points; la notion d'échelle est capitale. Je dois done préciser que, même dans une région encore insuffisamment connue sur le plan phyto-écologique, les études que nous entreprenons sont à l'échelle du massif forestier, c'est-à-dira du millier à quelques milliers d'hectares, oủ la diversité écologique est déjả fortement réduite. Par ailleurs, je dois reconnaître avoir été trop schématique en parlant d'approche purement floristique; en fait, nous utilisons les documents disponibles relatifs à la connaissance du milieu (tels que, le plus souvent, les cartes topographiques et géologiques) pour établir notre réseau de relevés : c'est la technique de l'échantillonnage « strati- 
fié " évoquée par M. Gooron. Je citerai comme exemple l'étude en cours du massif de Haye, où la densité de points dans les zones de vallons est quatre fois plus forte que sur le plateau.

M. AYmonin. - Il apparaît que la communication de M. BECKER a une grande portée théorique; mais elle met en relief les difficultés d'homogénéiser les vues du praticien et celles du chercheur qui essaie de replacer les faits dans un plan causal plus général.

Il convient de ne pas oublier que la signification d'une unité taxinomique n'est pas obligatoirement identique d'une région à une autre, sur le plan de l'écologie fine; indẻpendamment des races écologiques, des écomorphoses ou des écotypes, d'autres faits peuvent intervenir qui font que, par exemple, une espèce appartiendra aux groupements méso- ou subxérophiles dans une région " humide » et aux groupements de types hygrophiles dans une région " plus séche \# ou sur des sols à faible rétention en eau. On peut aussi évoquer le cas - dont l'étude est due d'ailleurs à M. BECKER - de la forêt de Charmes, qui a fourni un magnifique exemple de la « distorsion » qui peut exister en matière d'interprétation, si des faits historiques font que la phase actuelle du peuplement est en disjonction avec des phases passées, dont l'analyse pédologique (ou parfois la présence d'espèces insolites) nous laisse entrevoir l'existence.

C'est pourquoi il y a évidemment des difficultés dans la méthode floristique. Cependant, le spectre floristique d'une station est incontestablement, globalement, un indicateur précieux de cette station, essentiellement sur un plan régional. Si la considération de quelques espèces significatives ne permet pas toujours de définir totalement un milieu ou une station, ces espèces permettent néanmoins de cerner d'une manière très valable, et souvent très précise, un problème stationnel. II suffit de penser au tableau tout-à-fait simple et tout-à-fait efficace établi pour quelques plantes significatives (moins de 10) en forêt landaise par KUHNHOLTZLORDAT et LEREDDE. II est cependant très nécessaire de souligner que ces mêmes espèces, si elles gardent souvent une valeur écologique comparable dans d'autres régions, ne sauraient être immédiatement acceptées comme ayant strictement la même valeur sur des substrats différents, ayant eu une évolution pédologique différente. Là encore, comme le soulignait M. BECKER, l'arsenal des groupes écologiques dont nous pouvons disposer est à considérer en fonction d'une expérience régionale de la question.

En élargissant peut-être un peu le débat à propos de cet exposé qui évoque beaucoup de problèmes, il nous faut ajouter deux remarques. La première est que des groupes d'espéces significatives écologiquement vis-à-vis d'un milieu pris dans son ensemble, ne le sont pas obligatoirement d'une manière parallèle vis-à-vis d'un seul facteur. II serait aisé, en matière forestière, de souligner que la considération de la fertilité, par exemple, pourra conduire à des interprétations assez diverses selon que l'on s'adresse aux niveaux du sol utilisés par les arbres ou à ceux utilisés par les composantes des tapis herbacés. Mais cependant, là encore, l'examen du spectre floristique global, en fonction des affinités sociologiques ou écologiques des diverses espèces (phanérogames et cryptogames) de ce spectre, trompera rarement quant à l'interprétation de la situation écologique de la station considérée.

$\mathrm{La} 2^{e}$ remarque est d'un tout autre ordre, et aussi plus fondaməntale. On ne sait ce que réserve l'avenir, mais la dégradation des couvertures végétales du monde fait que l'on doit être vigilants quant à leur exploitation et à leur connaissance. Les travaux d'approche de la double définition de la station (strictement écologique d'une part, en fonction d'impératifs de production d'autre part), devraient permettre d'aboutir à une conception unitaire qui 
tiendrait compte des nécessités biologiques du milieu forestier et des besoins de l'Homme. Cette connaissance nous semble, personnellement, particulièrement essentielle sur le plan dynamique dans le cadre des équilibres biologiques naturels ou secondaires; il n'est pas vain d'envisager, à plus ou moins brève échéance, la nécessité de reconstituer, quand cela se pourra encore, les peuplements, forestiers ou autres, en équilibre avec les données écologiques régionales. Des expériences dans cette voie ont d'ailleurs été tentées depuis une dizaine d'année, dans la région de Stavropol en U.R.S.S., visant à reconstituer les bioconoses naturelles.

Ceci semble un des points pour lesquels des recherches telles celles qui viennent d'être exposées peuvent apporter, outre leur portée pratique, des éléments déterminants.

M. Decourt. - Pourquoi ne pas faire intervenir également, au moment de la description botanique (et non pas en fin d'étude), des indices de productivité définis sur le peuplement ligneux (la hauteur dominante par exemple), qui ont une valeur indéniable d'indicateurs ècologiques?

M. BECKER. - C'est effectivement ce que nous faisons dans la plupart de nos études, en mesurant, dès la première phase (floristique), la hauteur dominante, que nous corrigeons en fonction de l'âge du peuplement.

M. Marion. - Permettez-moi de vous exposer brièvement un type d'étude, mené en Tunisie avec, d'ailleurs, la collaboration de M. TimBaL. Le problème à résoudre était de préparer les périmètres à reboiser :

— les cartes de végétation au 1/200 000 ont permis d'implanter ces périmètres,

- pour la préparation de chaque périmètre, l'étude était faite à partir de la photo aérienne au 1/25000, et s'appuyait sur les espèces caractéristiques des principaux milieux écologiques favorables à telle ou telle mise en valeur (telle espèce de reboisement, maintien en l'état, aménagement sylvo-pastoral, etc.), ainsi que sur les facteurs pédologiques limitants,

- plutôt que d'établir un document cartographique, il nous a paru essentiel de reporter aussitôt sur le terrain les limites des milieux : ceci avec l'aide d'équipes spécialisées dans l'ouverture de layons, avec repères de couleurs à la peinture.

Ces deux phases de travail sont du ressort de techniciens dûment formés pour leur secteur, après préparation des données et de la méthodologie par l'institut de recherche appliquée.

M. Martinot-Lagarde, - Dans la pratique, ce que désire le forestier, en France, c'est une liste de plantes valables pour une région donnée et ayant une signification quant à la productivité et à la facilité de régénération naturelle; et ceci pour une ou plusieurs essences.

M. Grandjean. - 11 est en effet important d'essayer de définir une méthodologie simple pour la cartographie des stations en milieu forestier, en mettant à la disposition du gestionnaire des groupements écologiques même simplifiés, mais testés en relation avec la production ligneuse.

M. DUPIAS, - II doit être possible de fournir au praticien quelques critères floristiques simples permettant de distinguer des unités écologiques, au moins en première approximation. Le botaniste, après une étude fine des groupes écologiques, doit sélectionner les 
quelques espèces significatives des principaux caractères ècologiques (humidité du sol, fertilité...) et les plus facilement identifiables. Nous citerons comme exemple celui de la carte de la forêt landaise, où ont été sélectionnées cinq espèces seulement pour le gradient d'humidité, et trois pour la fertilité. L 'étude floristique fine doit donc permettre de dégager quelques " recettes " simples pour identifier la « station " au sens du forestier.

M. BECKER, - Ceci est effectivement souhaitable, et très souvent possible. Ainsi, en forêt communale de Charmes, et pour toutes les forêts similaires de la région, a-t-on pu proposer au gestionnaire cinq espéces seulement pour définir les principaux types de stations; par hydromorphie et dégradation décroissantes : Molinie, Carex brizoídes, Canche cespiteuse, Luzule blanchâtre et Poa chaixi. En forêt domaniale de Villers-Cotterêts, notre étude a montré qu'une quinzaine d'espèces sont également suffisantes pour classer rapidement les diverses stations; l'opération est d'ailleurs actuellement en cours, menée par les services de l'Office National des Forểts.

M. Lévy. - Peut-on vraiment, dans des forêts telles que celle de Charmes, utiliser la flore comme indice de productivité? L'intervention humaine a provoqué, dans certaines parties de la forêt, une remontée de la nappe, laquelle s'est traduite par une modification de la flore. Mais il n'est pas prouvé que si l'on effectuait, dans l'ensemble du massif, une coupe à blanc et l'on reboisait, par exemple en Épicéa, la production serait en rapport avec la répartition actuelle des espẻces herbacées, car alors les niveaux de nappe s'uniformiseraient peut-être.

M. BeCKer. - Le cas de la forêt de Charmes est en effet un peu particulier, en ce sens que l'accent a été mis sur l'aspect dynamique de la question : les espèces sociales observées aujourd'hui sont le reflet des conditions actuelles de milieu, qui peuvent évoluer, dans un sens ou dans l'autre, mais plus ou moins rapidement selon les facteurs écologiques : si l'apparition de l'évolution régressive est d'origine humaine, par action sur la structure du peuplement (celle-ci peut effectivement être rapidement corrigée), le processus s'est poursuivi par une dégradation des horizons superficiels du sol, de leurs propriétés physiques surtout, qui, elle, est beaucoup plus difficilement réversible, et qui a une répercussion certaine sur la productivité - au sens large - des stations. Par ailleurs, la nature de l'espèce sociale qui s'installe, à hydromorphie semblable — Carex ou Canche, Luzule ou Poa — est également le reflet des propriétés intrinsèques, plus permanentes, du milieu. Enfin, si notre attention a été canaliséc sur ces espéces sociales, n'oublions pas les résultats obtenus sur l'ensemble de la flore, et les groupes écologiques mis en évidence, plus discrets mais de valeur également plus permanente.

M. HUbert. - On nous demande souvent, en forêt privée, quelle est la vocation d'un terrain portant actuellement un peuplement sans valeur. Pour répondre à cette question, la flore à elle seule est probablement insuffisante, et peut conduire à des erreurs de diagnostic; on doit y adjoindre d'autres observations (pédologiques, climatiques...). Ce dont nous avons besoin, c'est surtout d'une méthode de travail et d'analyse du milieu qui permette de réduire les risques au moment d'un choix engageant l'avenir pour une longue période.

M. VIART, - J'estime également, pour ma part, que les forestiers de terrain doivent surtout attendre du C.N.R.F. des méthodes de travail, et non des « rečttes » pour régler leurs problèmes pratiques de gestion. 
M. JaCamon, - Le souci du Laboratoire de Botanique du C.N.R.F. est d'établir les relations liant la flore aux conditions écologiques, et de préciser ainsi des groupes écologiques indicateurs de la station. La connaissance de ces groupes n'empêchera pas l'observation pédologique, mais elle pourra l'orienter et, en général, la localiser.

Le, ou les groupes écologiques devront done figurer en bonne place sur les « fiches d'identité " des stations.

Quant à savoir si ces groupes pourront se traduire par des espèces faciles à reconnaitre, c'est une question de cas particuliers. Un effort sera toujours fait pour utiliser les végétaux ligneux, mieux connus et présents en toutes saisons. Mais la présence des arbres est influencée par l'action du sylviculteur, et certains ont une grande amplitude écologique (le Hêtre par exemple). Il faudra donc lcur ajouter quelques végétaux indicateurs, en s'efforçant de sortir ceux qui sont essentiels et qui constitueront le "groupe écologique simplifié ". Pour une région donnée, le nombre en sera suffisamment réduit pour qu'il soit possible au gestionnaire de les connaître, à quelque niveau technique qu'il se place.

Enfin, le C.N.R.F, ne pourra se situer qu'au plan des méthodes. Il apparaît indispensable que des équipes techniques (pédologie, phytosociologie), opérant toutes avec les mêmes méthodes, travaillent, au niveau des régions, et en liaison avec le C.N.R.F., à définir les « fiches d'identité » des stations (par étages géologiques par exemple). Les services de gestion pourront ensuite passer à la cartographie de leurs massifs.

M. VENET. - II est utile pour le praticien que le C.N.R.F. leur donne des cartes de stations, mais, effectivement, je ne pense pas qu'il puisse le faire pour toutes les forêts de France. Il apparait donc surtout nécessaire de fournir aux gestionnaires les moyens de faire eux-mèmes des cartes, en leur donnant des « fiches d'identité " des stations, avec des critères faciles à observer à l'échelle des unités de gestion forestières.

M. BOURGENOT. - Les forestiers sont extrêmement intéressés par ce qui a été dit aujourd'hui par divers conférenciers sur la station.

Ils ont en effet besoin, pour mieux aménager leurs forêts, d'y définir des types de peuplements susceptibles d'un même mode de traitement. Cette notion de « type de peuplement $n$ est moins précise que celle de "station ", mais elle en est voisine; du moins elle part de la notion analytique de station qui demeure un préalable.

Les forestiers sont donc fortement demandeurs pour qu'à l'issue de cette très intéressante réunion, les chercheurs, les scientifiques, se mettent d'accord pour une définition unique et pratique de la station. 